\title{
ON THE SECOND ORDER DERIVATIVE ESTIMATES FOR DEGENERATE PARABOLIC EQUATIONS
}

\author{
ILDOO KIM AND KYEONG-HUN KIM
}

AbSTRACT. We study the parabolic equation

$$
\begin{aligned}
& u_{t}(t, x)=a^{i j}(t) u_{x^{i}{ }^{j}}(t, x)+f(t, x), \quad(t, x) \in[0, T] \times \mathbf{R}^{d} \\
& u(0, x)=u_{0}(x)
\end{aligned}
$$

with the full degeneracy of the leading coefficients, that is,

$$
\left(a^{i j}(t)\right) \geq \delta(t) I_{d \times d} \geq 0 .
$$

It is well known that if $f$ and $u_{0}$ are not smooth enough, say $f \in \mathbb{L}_{p}(T):=$ $L_{p}\left([0, T] ; L_{p}\left(\mathbf{R}^{d}\right)\right)$ and $u_{0} \in L_{p}\left(\mathbf{R}^{d}\right)$, then in general the solution is only in $C\left([0, T] ; L_{p}\left(\mathbf{R}^{d}\right)\right)$, and thus derivative estimates are not possible.

In this article we prove that $u_{x x}(t, \cdot) \in L_{p}\left(\mathbf{R}^{d}\right)$ on the set $\{t: \delta(t)>0\}$ and

$$
\int_{0}^{T}\left\|u_{x x}(t)\right\|_{L_{p}}^{p} \delta(t) d t \leq N(d, p)\left(\int_{0}^{T}\|f(t)\|_{L_{p}}^{p} \delta^{1-p}(t) d t+\left\|u_{0}\right\|_{B_{p}^{2-2 / p}}^{p}\right),
$$

where $B_{p}^{2-2 / p}$ is the Besov space of order $2-2 / p$. We also prove that $u_{x x}(t, \cdot) \in$ $L_{p}\left(\mathbf{R}^{d}\right)$ for all $t>0$ and

$$
\int_{0}^{T}\left\|u_{x x}\right\|_{L_{p}\left(\mathbf{R}^{d}\right)}^{p} d t \leq N\left\|u_{0}\right\|_{B_{p}^{2-2 /(\beta p)}}^{p}
$$

if $f=0, \int_{0}^{t} \delta(s) d s>0$ for each $t>0$, and a certain asymptotic behavior of $\delta(t)$ holds near $t=0$ (see [1.3). Here $\beta>0$ is the constant related to the asymptotic behavior in 1.3. For instance, if $d=1$ and $a^{11}(t)=\delta(t)=$ $1+\sin (1 / t)$, then $(0.3)$ holds with $\beta=1$, which actually equals the maximal regularity of the heat equation $u_{t}=\Delta u$.

\section{INTRODUCTION}

The study of degenerate elliptic and parabolic equations was started long time ago. For instance, $L_{2}$-theory for the fully degenerate elliptic and parabolic equation was developed in [14, 15, 16, 17, and $L_{p}$-theory was introduced in 4, 10. In these articles, it is assumed that $A:=\left(a^{i j}\right) \geq 0$ and it depends both $t$ and $x$. Under the such general degeneracy, the solution is only in $C\left([0, T] ; L_{p}\right)$ and can not be

2010 Mathematics Subject Classification. 35K65, 35B65, 35K15.

Key words and phrases. Time degenerate parabolic equations, Maximal $L_{p}$-regularity, initialvalue problem.

The research of the first author was supported by Basic Science Research Program through the National Research Foundation of Korea(NRF) grant funded by the Korea government(2017R1C1B1002830).

The research of the second author was supported by Basic Science Research Program through the National Research Foundation of Korea(NRF) grant funded by the Korea government (2017R1D1A1B03033255). 
smoother than $f$ and $u_{0}$. This can be easily seen by taking $A=0$ so that

$$
u(t)=u(0)+\int_{0}^{t} f d s, \quad \forall t>0 .
$$

Thus, some extra conditions on the degeneracy are needed for better regularity of solutions. Especially there are many articles handling the degeneracy depending mainly on the space variable. See e.g. [18, 9, 7, 2] (analtyic methods) and [19, 3] (probabilistic method). These results focused on controlling the degeneracy of $a^{i j}(x)$ near the boundary of domains and used certain weights or considered splitting the boundary with a modified Dirichlet condition.

In this article we investigate a maximal regularity of solutions under the condition that the degeneracy depends only on the time variable, that is $a^{i j}=a^{i j}(t)$. Based on a standard perturbation argument one can extend our result to the general case $a^{i j}=a^{i j}(t, x)$ (see Remark 2.9). As mentioned in the abstract above, under condition (0.2), we prove that $u_{x x}(t) \in L_{p}$ on $\{t: A(t)>0\}$ and

$$
\left\|u_{x x}\right\|_{L_{p}\left([0, T], \delta(t) d t ; L_{p}\right)} \leq N(d, p)\left(\|f\|_{L_{p}\left([0, T], \delta^{1-p}(t) d t ; L_{p}\right)}+\left\|u_{0}\right\|_{B_{p}^{2-2 / p}}\right) .
$$

This might look absurd at first glance if one considers the above example (see (1.1)), but in such case (1.2) is trivial because the left hand side is zero.

To estimate $u_{x x}$ without the help of the weight $\delta(t) d t$, we further assume that $\int_{0}^{t} \delta(s) d s>0$ for any $t>0,\left|a^{i j}(t)\right| \leq N \delta(t)$, and for some $\beta, t_{0}, N_{0}>0$,

$$
\left|\left\{t \in\left[0, t_{0}\right]: h \leq \int_{0}^{t} \delta(s) d s<4 h\right\}\right| \leq N_{0} h^{1 / \beta}, \quad \forall h \approx 0 .
$$

Under these conditions we prove

$$
\int_{0}^{T}\left\|u_{x x}\right\|_{L_{p}}^{p} d t \leq N\left\|u_{0}\right\|_{B_{p}^{2\left(1-\frac{1}{\beta p}\right)}}^{p}, \quad \text { provided } f=0 .
$$

If, for instance,

$$
t^{\beta_{0}} \leq c \int_{0}^{t} \delta(s) d s, \quad \forall t>0
$$

for some $c, \beta_{0}>0$, then $\left\{t \in[0,1]: h \leq \int_{0}^{t} \delta(s) d s<4 h\right\} \subset\left[0,(4 h)^{1 / \beta_{0}}\right]$, and thus (1.3) holds with $\beta=\beta_{0}$. One can check that e.g. $\delta(t)=1+\sin (1 / t)$ satisfies (1.5) with $c=2$ and $\beta_{0}=1$ (see Examples 2.12 and 2.13 for detail and more examples).

Actually, (1.2) was already introduced in 8 if $\delta(t)=t^{\alpha}$, where $\alpha>-1$. Note that if $\delta(t)=t^{\alpha}$ then $\int_{0}^{t} \delta(s) d s=(1+\alpha)^{-1} t^{\alpha+1}$ and thus (1.5) holds with $\beta_{0}=\alpha+1$. We remark that our approach is somewhat different from the approach in [8]. If $\delta(t)=t^{\alpha}$ then equation (0.1) has uniform ellipticity on $\left[e^{-n-1}, e^{-n}\right]$ for each $n \geq 1$ since $t^{\alpha} \approx e^{-n \alpha}$. Using a classical result for equations with uniform ellipticity one can get some local estimates on such intervals, and combining local estimates one can derive (1.2). In this article, since our equation is not locally elliptic, we do not follow the idea in [8]. Instead, we use a certain approximation method. We first assume $\delta(t) \geq \varepsilon>0$ and prove (1.2) with constant $N(d, p)$ independent of $\varepsilon>0$, then we take $\varepsilon \rightarrow 0$ for the general case.

This paper is organized as follows. In Section 2 we introduce some function spaces and our main results, Theorem 2.7 and Theorem 2.14. Theorem 2.7 is proved in Section 3, and Theorem 2.14 is proved in Section 4.

We finish the introduction with notation used in the article. 
- We use Einstein's summation convention throughout this paper.

- $\mathbb{N}$ and $\mathbb{Z}$ denote the natural number system and the integer number system, respectively. As usual $\mathbf{R}^{d}$ stands for the Euclidean space of points $x=$ $\left(x^{1}, \ldots, x^{d}\right)$. For $i=1, \ldots, d$, multi-indices $\alpha=\left(\alpha_{1}, \ldots, \alpha_{d}\right), \alpha_{i} \in\{0,1,2, \ldots\}$, and functions $u(x)$ we set

$$
u_{x^{i}}=\frac{\partial u}{\partial x^{i}}=D_{i} u, \quad D^{\alpha} u=D_{1}^{\alpha_{1}} \cdot \ldots \cdot D_{d}^{\alpha_{d}} u .
$$

- $C^{\infty}\left(\mathbf{R}^{d}\right)$ denotes the space of infinitely differentiable functions on $\mathbf{R}^{d}$. $\mathcal{S}\left(\mathbf{R}^{d}\right)$ is the Schwartz space consisting of infinitely differentiable and rapidly decreasing functions on $\mathbf{R}^{d}$. By $C_{c}^{\infty}\left(\mathbf{R}^{d}\right)$, we denote the subspace of $C^{\infty}\left(\mathbf{R}^{d}\right)$ with the compact support.

- For $n \in \mathbb{N}$ and $\mathcal{O} \subset \mathbf{R}^{d}$ and a normed space $F$, by $C(\mathcal{O} ; F)$, we denote the space of all $F$-valued continuous functions $u$ on $\mathcal{O}$ having $|u|_{C}:=$ $\sup _{x \in O}|u(x)|_{F}<\infty$.

- For $p \in[1, \infty)$, a normed space $F$, and a measure space $(X, \mathcal{M}, \mu)$, by $L_{p}(X, \mathcal{M}, \mu ; F)$, we denote the space of all $F$-valued $\mathcal{M}^{\mu}$-measurable functions $u$ so that

$$
\|u\|_{L_{p}(X, \mathcal{M}, \mu ; F)}:=\left(\int_{X}\|u(x)\|_{F}^{p} \mu(d x)\right)^{1 / p}<\infty,
$$

where $\mathcal{M}^{\mu}$ denotes the completion of $\mathcal{M}$ with respect to the measure $\mu$. If there is no confusion for the given measure and $\sigma$-algebra, we usually omit them.

- For measurable set $\mathcal{O} \subset \mathbf{R}^{d},|\mathcal{O}|$ denotes the Lebesgue measure of $\mathcal{O}$.

- By $\mathcal{F}$ and $\mathcal{F}^{-1}$ we denote the d-dimensional Fourier transform and the inverse Fourier transform, respectively. That is, $\mathcal{F}[f](\xi):=\int_{\mathbf{R}^{d}} e^{-i x \cdot \xi} f(x) d x$ and $\mathcal{F}^{-1}[f](x):=\frac{1}{(2 \pi)^{d}} \int_{\mathbf{R}^{d}} e^{i \xi \cdot x} f(\xi) d \xi$.

- If we write $N=N(a, b, \cdots)$, this means that the constant $N$ depends only on $a, b, \cdots$.

\section{Setting and main Results}

Let $T \in(0, \infty)$ be a fixed time and $d \in \mathbb{N}$ be the space dimension.

Assumption 2.1. (i) The coefficients $a^{i j}(t)(i, j=1, \ldots, d)$ are measurable and bounded, that is there exists a constant $M>0$ such that

$$
\left|a^{i j}(t)\right| \leq M, \quad \forall t>0, i, j .
$$

(ii) There exists a nonnegative measurable function $\delta(t)$ such that

$$
0 \leq \delta(t)|\xi|^{2} \leq a^{i j}(t) \xi^{i} \xi^{j}, \quad \forall(t, \xi) \in(0, \infty) \times \mathbf{R}^{d} .
$$

Remark 2.2. (i) Since we may assume that $\left(a^{i j}(t)\right)$ is symmetric, we can take $\delta(t)$ as the smallest eigenvalue of $\left(a^{i j}(t)\right)$. If $d=1$ we take $\delta(t)=a^{11}(t)$.

(ii) $\delta(t)$ is bounded due to Assumption 2.1.

For $p \in(1, \infty)$ and $n=0,1,2, \cdots$, denote

$$
H_{p}^{n}=H_{p}^{n}\left(\mathbf{R}^{d}\right)=\left\{u: D^{\alpha} u \in L_{p}\left(\mathbf{R}^{d}\right),|\alpha| \leq n\right\},
$$

and in general for $n \in \mathbf{R}$, we write $u \in H_{p}^{n}$ iff

$$
\|u\|_{H_{p}^{n}}:=\left\|(1-\Delta)^{n / 2} u\right\|_{L_{p}}:=\left\|\mathcal{F}^{-1}\left[\left(1+|\xi|^{2}\right)^{n / 2} \mathcal{F}(u)(\xi)\right]\right\|_{L_{p}}<\infty .
$$


Define

and for $m \in \mathbf{R} \backslash 0$,

$$
\mathbb{H}_{p}^{n}(T)=L_{p}\left([0, T], d t ; H_{p}^{n}\right)
$$

$$
\mathbb{H}_{p}^{n}\left(T, \delta^{m}\right):=L_{p}\left([0, T], \delta^{m}(t) d t ; H_{p}^{n}\right),
$$

i.e.

$$
f \in \mathbb{H}_{p}^{n}\left(T, \delta^{m}\right) \quad \Leftrightarrow \quad\|f\|_{\mathbb{H}_{p}^{n}\left(T, \delta^{m}\right)}:=\left(\int_{0}^{T}\|f(t, \cdot)\|_{H_{p}^{n}}^{p} \delta^{m}(t) d t\right)^{1 / p}<\infty .
$$

Simply we put

$$
\mathbb{L}_{p}(T):=\mathbb{H}_{p}^{0}(T), \quad \mathbb{L}_{p}\left(T, \delta^{m}\right)=\mathbb{H}_{p}^{0}\left(T, \delta^{m}\right) .
$$

Remark 2.3. (i) Let $u \in \mathbb{H}_{p}^{n}\left(T, \delta^{m}\right)$ for some $m>0$. Then possibly $u(t, \cdot) \notin H_{p}^{n}$ on the set $\{t: \delta(t)=0\}$.

(ii) Since $\delta(t)$ is bounded, for any $m_{1} \geq m_{2}$,

$$
\mathbb{H}_{p}^{\gamma}\left(T, \delta^{m_{2}}\right) \subset \mathbb{H}_{p}^{\gamma}\left(T, \delta^{m_{1}}\right)
$$

and in particular

$$
\mathbb{H}_{p}^{2}(T) \subset \mathbb{H}_{p}^{2}(T, \delta) \quad \text { and } \quad \mathbb{L}_{p}\left(T, \delta^{1-p}\right) \subset \mathbb{L}_{p}(T)
$$

Definition 2.4. Let $u \in \mathbb{L}_{p}(T)$. We say that $u$ is a solution to equation (0.1) iff for any $\varphi \in C_{c}^{\infty}\left(\mathbf{R}^{d}\right)$ the equality

$$
(u(t, \cdot), \varphi)=\left(u_{0}, \varphi\right)+\int_{0}^{t}\left(a^{i j}(s) u(s, \cdot), \varphi_{x^{i} x^{j}}\right) d s+\int_{0}^{t}(f(s, \cdot), \varphi) d s
$$

holds for all $t \leq T$.

Here is a classical result for the fully degenerate parabolic equation. See e.g. [10, Theorem 3.1], where it is assumed that $p \in[2, \infty)$ to handle stochastic parabolic equations. But for the deterministic case the proof goes through for all $p \in(1, \infty)$.

Theorem 2.5. Let $p>1, T<\infty$ and Assumption 2.1 hold. Then for any $f \in$ $\mathbb{L}_{p}(T)$ and $u_{0} \in L_{p}$, equation (0.1) has a unique solution $u \in C\left([0, T] ; L_{p}\right)$, and for this solution

$$
\|u\|_{C\left([0, T] ; L_{p}\right)} \leq N(p, T, d)\left(\|f\|_{\mathbb{L}_{p}(T)}+\left\|u_{0}\right\|_{L_{p}}\right) .
$$

Furthermore, if $f \in \mathbb{H}_{p}^{n}(T)$ and $u_{0} \in H_{p}^{n}$ for some $n \in \mathbf{R}$, then $u \in C\left([0, T] ; H_{p}^{n}\right)$.

Remark 2.6. Note that in the above result, the regularity (or differentiability) of the solution is not better than that of $f$ and $u_{0}$.

To state our regularity condition for the initial data, we introduce the Besov space characterized by the Littlewood-Paley operator. See [1, Chapter 6] or [6], Chapter 6] for more details. Let $\Psi$ be a nonnegative function on $\mathbf{R}^{d}$ so that $\hat{\Psi} \in$ $C_{c}^{\infty}\left(B_{2}(0) \backslash B_{1 / 2}(0)\right)$ and

$$
\sum_{j \in \mathbb{Z}} \hat{\Psi}\left(2^{-j} \xi\right)=1, \quad \forall \xi \in \mathbf{R}^{d}
$$

where $B_{r}(0):=\left\{x \in \mathbf{R}^{d}:|x| \leq r\right\}$ and $\hat{\Psi}$ is the Fourier transform of $\Psi$. For a tempered distribution $u$, we define

$$
\Delta_{j} u(x):=\Delta_{j}^{\Psi} u(x):=\mathcal{F}^{-1}\left[\hat{\Psi}\left(2^{-j} \xi\right) \mathcal{F} u(\xi)\right](x)
$$


and

$$
S_{0}(u)(x)=\sum_{j=-\infty}^{0} \Delta_{j} u(x),
$$

where the convergence is understood in the sense of distributions. Due to (2.3),

$$
u(x)=S_{0}(u)(x)+\sum_{j=1}^{\infty} \Delta_{j} u(x) .
$$

The Besov space $B_{p}^{s}$ with the order $s$ and the exponent $p$ is the space of all tempered distributions $u$ such that

$$
\|u\|_{B_{p}^{s}}:=\left\|S_{0}(u)\right\|_{L_{p}}+\left(\sum_{j=1}^{\infty} 2^{s p j}\left\|\Delta_{j} u\right\|_{L_{p}}^{p}\right)^{1 / p}<\infty .
$$

Now we introduce our first result to equation (0.1). We control an $L_{p}$-norm of $u_{x x}$ with the help of the weight $\delta(t) d t$.

Theorem 2.7. Let $p>1, n \in \mathbf{R}$, and Assumption 2.1 hold. Then for all $u_{0} \in$ $B_{p}^{n+2-2 / p}$ and $f \in \mathbb{H}_{p}^{n}\left(T, \delta^{1-p}\right)$, there exists a unique solution $u \in C\left([0, T] ; H_{p}^{n}\right) \cap$ $\mathbb{H}_{p}^{n+2}(T, \delta)$ to the problem

$$
u_{t}=a^{i j}(t) u_{x^{i} x^{j}}+f, \quad t \in(0, T) ; \quad u(0, \cdot)=u_{0} .
$$

Furthermore, for this solution we have

$$
\left\|u_{x x}\right\|_{\mathbb{H}_{p}^{n}(T, \delta)} \leq N(d, p)\left(\|f\|_{\mathbb{H}_{p}^{n}\left(T, \delta^{1-p}\right)}+\left\|u_{0}\right\|_{B_{p}^{n+2-2 / p}}\right) .
$$

The proof of Theorem 2.7 is given in Section 3 (see Lemma 3.2).

Remark 2.8. (i) By (2.8), $u_{x x}(t, \cdot) \in L_{p}$ on the set $\{t: \delta(t)>0\}$.

(ii) We do not assume $\int_{0}^{T} \delta(s) d s>0$ in Theorem 2.7. In other words, the equation can be completely degenerate on $(0, T)$. However, if $\delta(t)=0$ for almost every $t \in(0, T)$, then (2.8) becomes trivial.

In the following remark we consider the general case having coefficients depending on $(t, x)$ and uniformly continuous in $x$.

Remark 2.9. For simplicity assume $u_{0}=0$. Let $\left(a^{i j}\right)=\left(a^{i j}(t, x)\right) \geq \delta(t) I_{d \times d}$. Then, using (2.8) and a standard freezing coefficients argument one can easily prove that there exists $\varepsilon_{0}>0$ such that (2.8) holds with $n=0$ if $\left|a^{i j}(t, x)-a^{i j}(t, y)\right| \leq$ $\varepsilon_{0} \delta(t)$ for all $x, y$ in the support of $u$.

Now assume that $\left|a^{i j}(t, x)\right| \leq N \delta(t)$ and $a^{i j}(t, x)$ are uniformly continuous in $x$ in the sense that for any $\varepsilon>0$, there exists $\kappa>0$, independent of $t$, such that

$$
|x-y|<\kappa \Rightarrow\left|a^{i j}(t, x)-a^{i j}(t, y)\right| \leq \varepsilon \delta(t) .
$$

Note that (2.9) holds if $a^{i j}(t, x)=a^{i j}(t) \eta(x)$, where $\eta(x)$ is nonnegative bounded uniformly continuous function.

Next we onsider an appropriate partition of unity $\left\{\eta^{n}: n \geq 1\right\}$ of $\mathbf{R}^{d}$ such that each $\eta^{n}$ has a support in a ball of radius $\kappa_{0}$ which corresponds to $\varepsilon_{0}$ in (2.9). Note that for each $n$,

$$
\left(u \eta^{n}\right)_{t}=a^{i j}\left(u \eta^{n}\right)_{x^{i} x^{j}}-2 a^{i j} u_{x^{i}} \eta_{x^{j}}^{n}-a^{i j} u \eta_{x^{i} x^{j}}^{n}+f \eta^{n}, \quad t>0 .
$$


Thus, by the choice of $\kappa_{0}$, for each $t \leq T$,

$$
\left\|\left(u \eta^{n}\right)_{x x}\right\|_{\mathbb{L}_{p}(t, \delta)}^{p} \leq N\left\|-2 a^{i j} u_{x^{i}} \eta_{x^{j}}^{n}-a^{i j} u \eta_{x^{i} x^{j}}^{n}+f \eta^{n}\right\|_{\mathbb{L}_{p}\left(t, \delta^{1-p}\right)}^{p} .
$$

Obviously,

$$
\left\|\eta^{n} u_{x x}\right\|_{\mathbb{L}_{p}(t, \delta)}^{p} \leq N\left(\left\|\left(u \eta^{n}\right)_{x x}\right\|_{\mathbb{L}_{p}(t, \delta)}^{p}+\left\|u_{x} \eta_{x}^{n}\right\|_{\mathbb{L}_{p}(t, \delta)}^{p}+\left\|u \eta_{x x}^{n}\right\|_{\mathbb{L}_{p}(t, \delta)}^{p}\right) .
$$

Thus, summing up these estimates with respect to $n$ and using (2.10) we get

$$
\begin{aligned}
\left\|u_{x x}\right\|_{\mathbb{L}_{p}(t, \delta)}^{p} & \leq N\left\|u_{x}\right\|_{\mathbb{L}_{p}(t, \delta)}^{p}+N\|u\|_{\mathbb{L}_{p}(t, \delta)}^{p}+N\left\|a^{i j} u_{x^{i}}\right\|_{\mathbb{L}_{p}\left(t, \delta^{1-p}\right)}^{p} \\
& +N\left\|a^{i j} u\right\|_{\mathbb{L}_{p}\left(t, \delta^{1-p}\right)}^{p}+N\|f\|_{\mathbb{L}_{p}\left(T, \delta^{1-p}\right)}^{p} .
\end{aligned}
$$

This and the assumption $\left|a^{i j}(t, x)\right| \leq N \delta(t)$ certainly lead to

$$
\left\|u_{x x}\right\|_{\mathbb{L}_{p}(t, \delta)}^{p} \leq N\left\|u_{x}\right\|_{\mathbb{L}_{p}(t, \delta)}^{p}+N\|u\|_{\mathbb{L}_{p}(t, \delta)}^{p}+N\|f\|_{\mathbb{L}_{p}\left(T, \delta^{1-p}\right)}^{p} .
$$

Furthermore, using inequalities

$$
\left\|u_{x}(s, \cdot)\right\|_{L_{p}} \leq \epsilon\left\|u_{x x}(s, \cdot)\right\|_{L_{p}}+\epsilon^{-1}\|u(s, \cdot)\|_{L_{p}}, \quad\|u\|_{\mathbb{L}_{p}(t, \delta)} \leq N\|u\|_{\mathbb{L}_{p}(t)},
$$

and taking $\epsilon$ sufficiently small, we get for each $t \leq T$

$$
\left\|u_{x x}\right\|_{\mathbb{L}_{p}(t, \delta)}^{p} \leq N\left(\|u\|_{\mathbb{L}_{p}(t)}^{p}+\|f\|_{\mathbb{L}_{p}\left(T, \delta^{1-p}\right)}^{p}\right) .
$$

Moreover, since $u$ is a solution to (2.7) and $u_{0}=0$, for all $s \leq t \leq T$, we get

$$
\begin{aligned}
\|u(s, \cdot)\|_{L_{p}}^{p} & =\left\|\int_{0}^{s} u_{r}(r, \cdot) d r\right\|_{L_{p}}^{p}=\left\|\int_{0}^{s}\left(a^{i j} u_{x^{i} x^{j}}+f\right)(r, \cdot) d r\right\|_{L_{p}}^{p} \\
& \leq N \int_{0}^{s}\left(\delta^{p}(r)\left\|u_{x x}(r, \cdot)\right\|_{L_{p}}^{p}+\|f(r, \cdot)\|_{L_{p}}^{p}\right) d r \\
& \leq N \int_{0}^{s}\left(\delta(r)\left\|u_{x x}(r, \cdot)\right\|_{L_{p}}^{p}+\|f(r, \cdot)\|_{L_{p}}^{p}\right) d r,
\end{aligned}
$$

where the last inequality is due to the assumption that $\delta(t)$ is bounded. Inequality (2.14) and integration on $[0, t]$ give

$$
\|u\|_{\mathbb{L}_{p}(t)}^{p} \leq N(T)\left(\int_{0}^{t}\left\|u_{x x}\right\|_{\mathbb{L}_{p}(s, \delta)}^{p} d s+\|f\|_{\mathbb{L}_{p}\left(T, \delta^{1-p}\right)}^{p}\right), \quad \forall t \leq T .
$$

From (2.13) and (2.15), we get, for any $t \leq T$,

$$
\begin{aligned}
\left\|u_{x x}\right\|_{\mathbb{L}_{p}(t, \delta)}^{p} & \leq N\|u\|_{\mathbb{L}_{p}(t)}^{p}+N\|f\|_{\mathbb{L}_{p}\left(T, \delta^{1-p}\right)}^{p} \\
& \leq N \int_{0}^{t}\left\|u_{x x}\right\|_{\mathbb{L}_{p}(s, \delta)}^{p} d s+N\|f\|_{\mathbb{L}_{p}\left(T, \delta^{1-p}\right)}^{p} .
\end{aligned}
$$

This and Gronwall's inequality lead to (2.8) with $n=0$.

Finally, we explain that one can slightly weaken the condition $\left|a^{i j}(t, x)\right| \leq N \delta(t)$ by

$$
\left|a^{i j}(t, x)\right| \leq N(\delta(t))^{\frac{2 p-1}{2 p}}, \quad p \geq \frac{3}{2} .
$$

Note that from (2.12) we can obtain

$$
\left\|u_{x}\right\|_{\mathbb{L}_{p}(t, \sqrt{\delta})} \leq \frac{1}{\epsilon}\|u\|_{\mathbb{L}_{p}(t)}+\epsilon\left\|u_{x x}\right\|_{\mathbb{L}_{p}(t, \delta)},
$$


where $\epsilon$ is a arbitrary positive constant. From (2.11) and (2.17),

$$
\begin{aligned}
\left\|u_{x x}\right\|_{\mathbb{L}_{p}(t, \delta)} \leq & N\left\|u_{x}\right\|_{\mathbb{L}_{p}(t, \delta)}+N\|u\|_{\mathbb{L}_{p}(t, \delta)}+N\left\|a^{i j} u_{x^{i}}\right\|_{\mathbb{L}_{p}\left(t, \delta^{1-p}\right)} \\
& +\left\|a^{i j} u\right\|_{\mathbb{L}_{p}\left(t, \delta^{1-p}\right)}+\|f\|_{\mathbb{L}_{p}\left(T, \delta^{1-p}\right)} \\
\leq & N\left\|u_{x}\right\|_{\mathbb{L}_{p}(t, \sqrt{\delta})}+N\|u\|_{\mathbb{L}_{p}(t)}+N\left\|u_{x}\right\|_{\mathbb{L}_{p}(t, \sqrt{\delta})}+\|u\|_{\mathbb{L}_{p}(t, \sqrt{\delta})} \\
& +\|f\|_{\mathbb{L}_{p}\left(T, \delta^{1-p}\right)} \\
\leq & N\left\|u_{x}\right\|_{\mathbb{L}_{p}(t, \sqrt{\delta})}+N\|u\|_{\mathbb{L}_{p}(t)}+\|f\|_{\mathbb{L}_{p}\left(T, \delta^{1-p}\right)} \\
\leq & \frac{N}{\epsilon}\|u\|_{\mathbb{L}_{p}(t)}+N \epsilon\left\|u_{x x}\right\|_{\mathbb{L}_{p}(t, \delta)}+N\|u\|_{\mathbb{L}_{p}(t)}+\|f\|_{\mathbb{L}_{p}\left(T, \delta^{1-p}\right)} .
\end{aligned}
$$

Taking $\epsilon$ sufficiently small, we get (2.13) again. Moreover, following (2.14), we have

$$
\begin{aligned}
\|u(s, \cdot)\|_{L_{p}}^{p} & =\left\|\int_{0}^{s} u_{r}(r, \cdot) d r\right\|_{L_{p}}^{p} \\
& \leq N \int_{0}^{s}\left(\delta \frac{2 p-1}{2}(r)\left\|u_{x x}(r, \cdot)\right\|_{L_{p}}^{p}+\|f(r, \cdot)\|_{L_{p}}^{p}\right) d r \\
& \leq N \int_{0}^{s}\left(\delta(r)\left\|u_{x x}(r, \cdot)\right\|_{L_{p}}^{p}+\|f(r, \cdot)\|_{L_{p}}^{p}\right) d r
\end{aligned}
$$

Therefore (2.16) is obtained again.

In Assumption 2.10 below we assume certain asymptotic behavior of $\delta(t)$ near $t=0$ to obtain

$$
\|u\|_{\mathbb{L}_{p}(T)}+\left\|u_{x x}\right\|_{\mathbb{L}_{p}(T)} \leq C\left\|u_{0}\right\|_{B_{p}^{s}},
$$

where $s<2$ and $u$ is the solution to equation (0.1) with $f=0$. Obviously, 2.21) is impossible if $\delta(t)$ completely vanishes near $t=0$. Indeed, if $a^{i j}(t) \equiv 0$ near $t=0$ then we get $u=u_{0}$ near $t=0$.

Assumption 2.10. (i) $\delta(t)$ does not completely vanish near $t=0$. In other words, $\int_{0}^{t} \delta(s) d s>0$ for all $t>0$.

(ii) There exist $t_{0} \in(0, T), \beta>0$, and $N_{0}>0$ such that for all $h>0$,

$$
\left|\left\{t \in\left[0, t_{0}\right]: h \leq \int_{0}^{t} \delta(s) d s<4 h\right\}\right| \leq N_{0} h^{1 / \beta}
$$

(iii) There exists a constant $\bar{N}_{0}>0$ such that

$$
\left|a^{i j}(t)\right| \leq \bar{N}_{0} \delta(t) \quad \forall t>0 .
$$

Remark 2.11. (i) If $d=1$ then we can take $\delta(t)=a^{11}(t)$, and thus 2.23) holds with $\bar{N}_{0}=1$.

(ii) If (2.22) holds with some $t_{0}>0$, then it also holds for any $t_{0}^{\prime}<t_{0}$ and therefore we may assume that $t_{0}$ is very small. In particular, we put $t_{0}<1 \wedge T$.

(iii) Since $t_{0}$ can be taken very small and $\int_{0}^{t} \delta(s) d s$ goes to zero as $t \rightarrow 0$, it is sufficient that (2.22) holds only for all sufficiently small $h>0$.

(iv) Obviously, if $\delta(t)>c>0$ near $t=0$ then (2.22) holds with $\beta=1$.

Here are two examples related to Assumption 2.10. 
Example 2.12 (Functions with weak scaling property). Assume that there exist constants $\alpha>-1, t_{0}>0$, and $N>0$ such that

$$
N t^{\alpha} \leq \delta(t) \quad \forall t \in\left(0, t_{0}\right) .
$$

Then Assumption 2.10(ii) holds with $\beta=\alpha+1$. Indeed,

$$
\begin{aligned}
\left|\left\{t \in\left[0, t_{0}\right]: h \leq \int_{0}^{t} \delta(s) d s<4 h\right\}\right| & \leq\left|\left\{t \in\left[0, t_{0}\right]: \frac{N}{\alpha+1} t^{\alpha+1}<4 h\right\}\right| \\
& \leq N_{0}(N, \alpha) h^{1 /(\alpha+1)} .
\end{aligned}
$$

(2.24) holds if $\delta(t)(\geq 0)$ is a polynomial or analytic near zero. Here are other (Bernstein) functions satisfying (2.24):

(1) $\delta(t)=\sum_{i=1}^{n} t^{\alpha_{i}}, 0<\alpha_{i}<1$

(2) $\delta(t)=\left(t+t^{\alpha}\right)^{\beta}, \alpha, \beta \in(0,1)$;

(3) $\delta(t)=t^{\alpha}(\log (1+t))^{\beta}, \alpha \in(0,1), \beta \in(0,1-\alpha)$;

(4) $\delta(t)=t^{\alpha}(\log (1+t))^{-\beta}, \alpha \in(0,1), \beta \in(0, \alpha)$;

(5) $\delta(t)=(\log (\cosh (\sqrt{t})))^{\alpha}, \alpha \in(0,1)$;

(6) $\delta(t)=(\log (\sinh (\sqrt{t}))-\log \sqrt{t})^{\alpha}, \alpha \in(0,1)$.

Example 2.13 (Oscillatory functions). Assume that there exist constants $\beta_{0}>0$, $t_{0}>0$, and $N>0$ such that

$$
N t^{\beta_{0}} \leq \int_{0}^{t} \delta(s) d s \quad \forall t \in\left(0, t_{0}\right) .
$$

Then by the argument in the previous example, $\delta(t)$ satisfies Assumption 2.10(ii) with $\beta=\beta_{0}$. Condition (2.25) is a generalization of (2.24) and is satisfied by lots of interesting oscillatory functions. For example, put

$$
\delta(t)=1+\sin (1 / t) .
$$

Note that $\delta(t)$ vanishes infinitely many times near $t=0$, and surprisingly (2.25) holds with $\beta_{0}=1$. This is because for any small $t>0$,

$$
\left|A_{t}\right|:=|\{s \leq t: \sin (1 / s) \geq-1 / 2\}| \geq t / 2,
$$

and therefore $\int_{0}^{t}(1+\sin (1 / s)) d s \geq \int_{A_{t}} 1 / 2 d s \geq t / 4$.

Theorem 2.14. Let $p>1$ and $T<\infty$. Suppose that Assumptions 2.1 and 2.10 hold. Then, for any $u_{0} \in B_{p}^{2(1-1 /(\beta p))}$, there exists a unique solution $u \in$ $C\left([0, T] ; L_{p}\right) \cap \mathbb{H}_{p}^{2}(T)$ to the problem

$$
u_{t}=a^{i j}(t) u_{x^{i} x^{j}}, \quad t \in(0, T) ; \quad u(0, \cdot)=u_{0},
$$

and for this solution we have

$$
\left\|u_{x x}\right\|_{\mathbb{L}_{p}(T)} \leq N\left\|u_{0}\right\|_{B_{p}^{2(1-1 /(\beta p))}},
$$

where $N$ is a constant depending only on $d, p, T, N_{0}, \bar{N}_{0}, \beta$, and $\int_{0}^{t_{0}} \delta(s) d s$.

The proof of this theorem will be given in Section 4 .

We remark that unlike (2.8), estimate (2.26) control $u_{x x}$ without the help of the weight $\delta(t) d t$. 
Remark 2.15. If $a^{i j}(t)=\delta^{i j}$, where $\delta^{i j}$ is the Kronecker delta, then (2.26) is a classical result for the heat equation. In this case, the constant $N$ in (2.26) depends only on $d$ and $p$, and in particular it is independent of $T$. This can be easily checked by the standard scaling argument (in homogeneous Besov space).

Example 2.13 and Theorem 2.14 yield the following result.

Corollary 2.16. Let $p \in(1, \infty), T \in(0, \infty), u_{0} \in B_{p}^{2-2 / p}$, and $d=1$. Then there exists a unique solution $u \in \mathbb{H}_{p}^{2}(T)$ to the equation

$$
u_{t}=(1+\sin (1 / t)) u_{x x}, \quad t>0 ; \quad u(0, \cdot)=u_{0},
$$

and we have

$$
\|u\|_{\mathbb{H}_{p}^{2}(T)} \leq N\left\|u_{0}\right\|_{B_{p}^{2(1-1 / p)}}
$$

Recall that (2.28) is the maximal regularity of the solution to the heat equation $u_{t}=u_{x x}$. Thus, the instant smoothing effect (or regularity of solution) of degenerate equation (2.27) is not affected at all by the degeneracy of the leading coefficient.

\section{Proof of Theorem 2.7}

Denote $A(t):=\left(a^{i j}(t)\right)$. Recall in Theorem 2.7, we only assume

$$
0 \leq \delta(t) I_{d \times d} \leq A(t), \quad\left|a^{i j}(t)\right| \leq M .
$$

Lemma 3.1. Let $\delta(t) \geq \varepsilon>0, u_{0} \in B_{p}^{2(1-1 / p)}, f \in \mathbb{L}_{p}(T)$, and $u \in \mathbb{L}_{p}(T)$ be a solution to problem (2.7), that is

$$
u_{t}=a^{i j} u_{x^{i} x^{j}}+f, \quad t \in(0, T) ; \quad u(0, \cdot)=u_{0} .
$$

Then

$$
\left\|u_{x x}\right\|_{\mathbb{L}_{p}(T)} \leq N(d, p)\left(\varepsilon^{-1}\|f\|_{\mathbb{L}_{p}(T)}+\left\|u_{0}\right\|_{B_{p}^{2(1-1 / p)}}\right) .
$$

The key point of Lemma 3.1 is that the constant $N(d, p)$ in (3.1) is independent of $T$ and $M$. Lemma 3.1 might be a very well known result, but we provide a (probabilistic) proof for the sake of the completeness.

Proof. We follow the idea in the proof of [13, Theorem 2.2]. Let $W_{t}=\left(W_{t}^{1}, \cdots, W_{t}^{d}\right)$ be a $d$-dimensional Wiener process on a probability space $(\Omega, \mathcal{F}, P)$. Since $A(t)$ is a nonnegative symmetric matrix, there exists a nonnegative symmetric matrix $\sigma(t)=\left(\sigma^{i j}(t)\right)$ such that

$$
2 A(t)=\sigma^{2}(t)
$$

We define

$$
X_{t}:=\int_{0}^{t} \sigma(t) d W_{t}, \quad\left(i . e ., X_{t}^{i}=\sum_{k=1}^{d} \int_{0}^{t} \sigma^{i k}(s) d W_{s}^{k},(i=1,2, \cdots, d)\right) .
$$

It is well known (see e.g. [13]) that the solution to equation (2.7) is given by

$$
u(t, x)=\mathbb{E}\left[u_{0}\left(x+X_{t}\right)\right]+\int_{0}^{t} \mathbb{E}\left[f\left(s, x+X_{t}-X_{s}\right] d s .\right.
$$


In particular if $A(t)=\varepsilon I_{d \times d}$, where $I_{d \times d}$ is the $d \times d$ identity matrix and $\varepsilon>0$, then $X_{t}=\sqrt{2 \varepsilon} W_{t}$, and

$$
u(t, x)=\mathbb{E}\left[u_{0}\left(x+\sqrt{2 \varepsilon} W_{t}\right)\right]+\int_{0}^{t} \mathbb{E}\left[f\left(s, x+\sqrt{2 \varepsilon} W_{t}-\sqrt{2 \varepsilon} W_{s}\right] d s .\right.
$$

Step 1. Assume $\delta(t)=\varepsilon$ and $A(t)=\varepsilon I_{d \times d}$. Then $v(t, x)=u\left(\varepsilon^{-1} t, x\right)$ satisfies the heat equation

$$
v_{t}(t, x)=\Delta v(t, x)+\varepsilon^{-1} f\left(\varepsilon^{-1} t, x\right) .
$$

From the classical result for the heat equation (cf. Remark 2.15), it follows that

$$
\left\|u_{x x}\right\|_{\mathbb{L}_{p}(T)} \leq N(d, p)\left(\varepsilon^{-1}\|f\|_{\mathbb{L}_{p}(T)}+\left\|u_{0}\right\|_{B_{p}^{2(1-1 / p)}}\right),
$$

and equivalently, we get

$$
\begin{aligned}
& \| D_{x}^{2}\left[\mathbb{E}\left[u_{0}\left(x+\sqrt{2 \varepsilon} W_{t}\right)\right]+\int_{0}^{t} \mathbb{E}\left[f\left(s, x+\sqrt{2 \varepsilon} W_{t}-\sqrt{2 \varepsilon} W_{s}\right] d s\right] \|_{\mathbb{L}_{p}(T)}\right. \\
& \leq N(d, p)\left(\varepsilon^{-1}\|f\|_{\mathbb{L}_{p}(T)}+\left\|u_{0}\right\|_{B_{p}^{2(1-1 / p)}}\right) .
\end{aligned}
$$

Step 2. General case. Write

$$
A(t)=\left(A(t)-\frac{\varepsilon}{2} I_{d \times d}\right)+\frac{\varepsilon}{2} I_{d \times d}=: \bar{A}(t)+\frac{\varepsilon}{2} I_{d \times d} .
$$

Let $\bar{W}_{t}$ be a $d$-dimensional Winer process which is independent of $W_{t}$ on a probability space $(\Omega, \mathcal{F}, P)$. Denote

$$
Y_{t}=\sqrt{\varepsilon} W_{t}, \quad \bar{\sigma}(t)=\sqrt{2 \bar{A}(t)}, \quad Z_{t}=\int_{0}^{t} \bar{\sigma}_{s} d \bar{W}_{s} .
$$

Then it is easy to show that for $0 \leq s<t, X_{t}-X_{s}$ and $Y_{t}+Z_{t}-Y_{s}-Z_{s}$ have the same probability distribution. Indeed, it suffices to check that the characteristic functions of two random variables coincide (cf. [11, Theorem 1.4.12]) and for any $\xi \in \mathbf{R}^{d}$, we get

$$
\begin{aligned}
& \mathbb{E} e^{i \xi \cdot\left(Y_{t}-Y_{s}+Z_{t}-Z_{s}\right)}=\mathbb{E} e^{i \xi \cdot\left(Y_{t}-Y_{s}\right)} \mathbb{E} e^{i \xi \cdot\left(Z_{t}-Z_{s}\right)}=e^{-\varepsilon(t-s)|\xi|^{2}} \mathbb{E} e^{i \xi \cdot \int_{s}^{t} \bar{\sigma}_{s} d \bar{W}_{r}} \\
= & e^{-\varepsilon(t-s)|\xi|^{2}} e^{-\int_{s}^{t}\left|\bar{\sigma}_{r} \xi\right|^{2} d r}=e^{-\int_{s}^{t}\left|\sigma_{r} \xi\right|^{2} d r}=\mathbb{E} e^{i \xi \cdot \int_{s}^{t} \sigma_{r} d W_{r}} .
\end{aligned}
$$

The third and fifth equalities above are trivial if $\bar{\sigma}_{t}$ is a simple function, and the general case is obtained based on a standard approximation.

From (3.3) it follows that (recall that $Y$ and $Z$ are independent)

$$
\begin{aligned}
D_{x}^{2} u(t, x)= & D_{x}^{2}\left(\mathbb{E}\left[u_{0}\left(x+Y_{t}+Z_{t}\right)\right]+\int_{0}^{t} \mathbb{E}\left[f\left(s, x+Y_{t}-Y_{s}+Z_{t}-Z_{s}\right)\right] d s\right) \\
= & D_{x}^{2}\left(\mathbb{E}^{\prime}\left(\mathbb{E}\left[u_{0}\left(x+Y_{t}(\omega)+Z_{t}\left(\omega^{\prime}\right)\right)\right]\right)\right) \\
& +D_{x}^{2}\left(\int_{0}^{t} \mathbb{E}^{\prime}\left(\mathbb{E}\left[f\left(s, x+Y_{t}(\omega)-Y_{s}(\omega)+Z_{t}\left(\omega^{\prime}\right)-Z_{s}\left(\omega^{\prime}\right)\right)\right]\right) d s\right),
\end{aligned}
$$

where $\mathbb{E}$ and $\mathbb{E}^{\prime}$ denote the expectations with respect to $\omega$ and $\omega^{\prime}$, respectively. For each $\omega^{\prime} \in \Omega$, denote

$$
f_{Z\left(\omega^{\prime}\right)}(s, x)=f\left(s, x-Z_{s}\left(\omega^{\prime}\right)\right) .
$$


Then,

$$
\begin{aligned}
D_{x}^{2} u(t, x)= & \mathbb{E}^{\prime}\left[D_{x}^{2}\left(\mathbb{E}\left[u_{0}\left(x+\sqrt{\varepsilon} W_{t}(\omega)+Z_{t}\left(\omega^{\prime}\right)\right)\right]\right)\right] \\
& +\mathbb{E}^{\prime}\left[D_{x}^{2}\left(\int_{0}^{t} \mathbb{E}\left[f_{Z\left(\omega^{\prime}\right)}\left(s, x+\sqrt{\varepsilon} W_{t}(\omega)-\sqrt{\varepsilon} W_{s}(\omega)+Z_{t}\left(\omega^{\prime}\right)\right)\right] d s\right)\right] .
\end{aligned}
$$

Finally, since the $L_{p}\left(\mathbf{R}^{d}\right)$-norm is translation invariant, by (3.4) we get

$$
\begin{aligned}
&\left\|D_{x}^{2} u\right\|_{\mathbb{L}_{p}(T)}^{p} \\
& \leq \mathbb{E}^{\prime} {\left[\| D_{x}^{2}\left(\mathbb{E}\left[u_{0}\left(x+\sqrt{\varepsilon} W_{t}(\omega)+Z_{t}\left(\omega^{\prime}\right)\right)\right]\right)\right.} \\
&\left.+D_{x}^{2}\left(\mathbb{E} \int_{0}^{t} f_{Z\left(\omega^{\prime}\right)}\left(s, x+\sqrt{\varepsilon} W_{t}(\omega)-\sqrt{\varepsilon} W_{s}(\omega)+Z_{t}\left(\omega^{\prime}\right)\right) d s\right) \|_{\mathbb{L}_{p}(T)}^{p}\right] \\
&=\mathbb{E}^{\prime} {\left[\| D_{x}^{2}\left(\mathbb{E}\left[u_{0}\left(x+\sqrt{\varepsilon} W_{t}(\omega)\right)\right]\right)\right.} \\
&\left.+D_{x}^{2}\left[\mathbb{E} \int_{0}^{t} f_{Z\left(\omega^{\prime}\right)}\left(s, x+\sqrt{\varepsilon} W_{t}(\omega)-\sqrt{\varepsilon} W_{s}(\omega)\right) d s\right] \|_{\mathbb{L}_{p}(T)}^{p}\right] \\
& \leq N(d, p) \mathbb{E}^{\prime}\left[\varepsilon^{-1}\left\|f_{Z\left(\omega^{\prime}\right)}\right\|_{\mathbb{L}_{p}(T)}^{p}+\left\|u_{0}\right\|_{B_{p}^{2(1-1 / p)}}\right] \\
&=N(d, p)\left[\varepsilon^{-1}\|f\|_{\mathbb{L}_{p}(T)}^{p}+\left\|u_{0}\right\|_{B_{p}^{2(1-1 / p)}}\right] .
\end{aligned}
$$

The lemma is proved.

Note that to prove Theorem 2.7 it is enough to assume $n=0$. Thus we only need to prove the following.

Lemma 3.2. Let $p>1, T<\infty, f \in \mathbb{L}_{p}\left(T, \delta^{1-p}\right)$, and $u \in C\left([0, T] ; L_{p}\right)$ be a solution to equation 2.7). Then

$$
\int_{0}^{T}\left\|u_{x x}(t)\right\|_{L_{p}}^{p} \delta(t) d t \leq N(p, d)\left(\int_{0}^{T}\|f(t)\|_{L_{p}}^{p} \delta^{1-p}(t) d t+\left\|u_{0}\right\|_{B_{p}^{2(1-1 / p)}}^{p}\right) .
$$

Proof. Step 1. In this step, we assume $\delta(t) \geq \varepsilon>0$. Then

$$
\int_{0}^{\infty} \delta(t) d t=\infty, \quad \text { and } \quad \delta^{-1} \in L_{1}([0, T])
$$

Denote

$$
\beta(t)=\int_{0}^{t} \delta(s) d s
$$

and let $\phi(t)$ be the inverse of $\beta(t)$, which is well defined for $t \in[0, \infty)$ due to $\delta(t)>\varepsilon>0$. Then, since $\beta^{\prime}(t)>0, \phi$ is differentiable everywhere and

$$
\phi^{\prime}(t)=\frac{1}{\beta^{\prime}(\phi(t))}=\frac{1}{\delta(\phi(t))} \leq \varepsilon^{-1} .
$$

Thus, in particular, $\phi$ is absolutely continuous. Define

$$
v(t, x)=u(\phi(t), x) .
$$


Note that $v$ satisfies

$$
v_{t}=\tilde{a}^{i j}(t) v_{x^{i} x^{j}}+\tilde{f}, \quad v(0, \cdot)=u_{0}
$$

where $\tilde{f}(t, x)=f(\phi(t), x) \phi^{\prime}(t)$ and

$$
\tilde{a}^{i j}(t):=a^{i j}(\phi(t)) \phi^{\prime}(t)=\frac{1}{\delta(\phi(t))} a^{i j}(\phi(t)) \geq I_{d \times d} .
$$

Thus by Lemma 3.1 for any $T_{0}>0$ such that $\phi\left(T_{0}\right) \leq T$, we have

$$
\left\|v_{x x}\right\|_{\mathbb{L}_{p}\left(T_{0}\right)} \leq N(d, p)\left(\|\tilde{f}\|_{\mathbb{L}_{p}\left(T_{0}\right)}+\left\|u_{0}\right\|_{B_{p}^{2(1-1 / p)}}\right) .
$$

Taking $T_{0}>0$ so that $\phi\left(T_{0}\right)=T$, we have

$$
\int_{0}^{T}\left\|u_{x x}\right\|_{L_{p}}^{p} \delta(t) d t \leq N(d, p)\left(\int_{0}^{T}\left\|\delta^{-1}(t) f\right\|_{L_{p}}^{p} \delta(t) d t+\left\|u_{0}\right\|_{B_{p}^{2(1-1 / p)}}\right) .
$$
2.5 .

Step 2. Let $\delta(t) \geq 0$, and assume $f \in \mathbb{H}_{p}^{2}(T)$ and $u_{0} \in H_{p}^{2}$. Then by Theorem

Denote

$$
u \in \mathbb{H}_{p}^{2}(T)
$$

Then

$$
A_{\varepsilon}(t)=A(t)+\varepsilon I, \quad \delta_{\varepsilon}(t):=\delta(t)+\varepsilon
$$

$u_{t}=a_{\varepsilon}^{i j} u_{x^{i} x^{j}}+f-\varepsilon \Delta u, \quad u(0, \cdot)=u_{0}$.

By Step 1 and the fact that $\delta_{\varepsilon}^{-p+1} \leq \delta^{-p+1}$,

$$
\begin{aligned}
& \int_{0}^{T}\left\|u_{x x}\right\|^{p} \delta_{\varepsilon}(t) d t \\
& \leq N(p, d)\left(\int_{0}^{T}\left\|\delta_{\varepsilon}^{-1}(f-\varepsilon \Delta u)\right\|_{L_{p}}^{p} \delta_{\varepsilon}(t) d t+\left\|u_{0}\right\|_{B_{p}^{2(1-1 / p)}}\right) \\
& \leq N(p, d)\left(\int_{0}^{T}\left\|\delta^{-1} f\right\|_{L_{p}}^{p} \delta(t) d t+\left\|u_{0}\right\|_{B_{p}^{2(1-1 / p)}}\right)+N(p, d) \int_{0}^{T}\left\|\delta_{\varepsilon}^{-1} \varepsilon \Delta u\right\|_{L_{p}}^{p} \delta_{\varepsilon}(t) d t .
\end{aligned}
$$

Note that $\frac{\varepsilon^{p}}{\delta_{\varepsilon}^{p}} \delta_{\varepsilon}$ is bounded above by $\delta+\varepsilon$ and goes to zero as $\varepsilon \rightarrow 0$. This is because if $\delta(t)=0$ then $\frac{\varepsilon^{p}}{\delta_{\varepsilon}^{p}} \delta_{\varepsilon}=\varepsilon$. Moreover, $\Delta u \in \mathbb{L}_{p}(T)$ due to (3.6). Therefore, by the dominated convergence theorem and the inequality $\delta \leq \delta_{\varepsilon}$, we get

$$
\int_{0}^{T}\left\|u_{x x}\right\|^{p} \delta(t) d t \leq N(p, d)\left(\int_{0}^{T}\left\|\delta^{-1} f\right\|_{L_{p}}^{p} \delta(t) d t+\left\|u_{0}\right\|_{B_{p}^{2(1-1 / p)}}\right) .
$$

Step 3. In general, we consider a mollification with respect to the space variable.

$$
u_{t}^{\varepsilon}=a^{i j}(t) u_{x^{i} x^{j}}^{\varepsilon}+f^{\varepsilon}, \quad u^{\varepsilon}(0, \cdot)=u_{0}^{\varepsilon}
$$

where

$$
u^{\varepsilon}(t, x)=\varepsilon^{-d} \int_{\mathbf{R}^{d}} u(t, x-y) \varphi\left(\varepsilon^{-1} y\right) d y
$$

and $\varphi \in C_{c}^{\infty}\left(\mathbf{R}^{d}\right)$ with the unit integral. Then $f^{\varepsilon} \in \mathbb{H}_{p}^{2}(T)$ and $u_{0}^{\varepsilon} \in H_{p}^{2}$, and by Theorem $\left[2.5 u^{\varepsilon} \in \mathbb{H}_{p}^{2}(T)\right.$. By Step 2, it follows that $u^{\varepsilon}$ is a Cauchy sequence in $L_{p}\left([0, T], \delta(t) d t ; H_{p}^{2}\right)$. The limit $u$ certainly satisfies the equation and estimate (3.7) also holds for $u$. The lemma is proved. 


\section{Proof of Theorem 2.14}

Recall that in Theorem 2.14 we assume

$$
\int_{0}^{t} \delta(s) d s>0, \quad \forall t>0 .
$$

By taking the Fourier transform to the equation

$$
u_{t}=a^{i j}(t) u_{x^{i} x^{j}}, \quad t \in(0, T) ; \quad u(0, \cdot)=u_{0},
$$

we get (at least formally)

$$
\mathcal{F}[u(t, \cdot)](\xi)=\exp \left(-\int_{0}^{t} a^{i j}(r) d r \xi^{i} \xi^{j}\right) \mathcal{F}\left[u_{0}\right](\xi),
$$

and the inverse Fourier transform gives

$$
u(t, x)=\int_{\mathbf{R}^{d}} p(t, x-y) u_{0}(y) d y=p(t, \cdot) * u_{0}(x)=: \mathcal{T}_{t} u_{0}(x),
$$

where

$$
p(t, x)=\mathcal{F}^{-1}\left[\exp \left(-\int_{0}^{t} a^{i j}(r) d r \xi^{i} \xi^{j}\right)\right](x) .
$$

Note that $p(t, x)$ is well defined since $\int_{0}^{t} \delta(s) d s>0$ and

$$
\left|\exp \left(-\int_{0}^{t} a^{i j}(r) d r \xi^{i} \xi^{j}\right)\right| \leq \exp \left(-\int_{0}^{t} \delta(r) d r|\xi|^{2}\right) .
$$

It is easy to check that the representation formula (4.2) gives the unique solution $u \in \mathbb{L}_{p}(T)$ to equation (4.1) if $u_{0} \in \mathcal{S}\left(\mathbf{R}^{d}\right)$, and the general case also holds due to a standard approximation argument based on estimate (2.2), where $\mathcal{S}\left(\mathbf{R}^{d}\right)$ denotes the Schwartz space on $\mathbf{R}^{d}$.

As in (2.4), define

$$
p_{j}(t, x):=\Delta_{j} p(t, x):=\Delta_{j} p(t, \cdot)(x):=\mathcal{F}^{-1}\left[\hat{\Psi}\left(2^{-j} \xi\right) \mathcal{F}[p(t, \cdot)](\xi)\right](x) .
$$

Lemma 4.1. Let $k \in \mathbb{Z}$ and $\gamma \in \mathbf{R}$. Suppose that Assumptions[2.1(ii) and 2.10(iii) hold. Then for all $t>0$

$$
\left\|\Delta^{\gamma / 2} p_{k}(t, \cdot)\right\|_{L_{1}} \leq N 2^{k \gamma} \exp \left(-c \int_{0}^{t} \delta(s) d s \cdot 2^{2 k}\right),
$$

where $c$ and $N$ are some positive constants depending only on $d, \gamma$, and $\bar{N}_{0}$.

Proof. Denote

$$
q_{k}(t, x)=\mathcal{F}^{-1}\left[|\xi|^{\gamma} \hat{\Psi}(\xi) \exp \left(-\int_{0}^{t} a^{i j}(s) d s \cdot 2^{2 k} \xi^{i} \xi^{j}\right)\right](x),
$$

and

$$
\hat{q}_{k}(t, \xi)=\mathcal{F}\left[q_{k}(t, \cdot)\right](\xi) .
$$

Then considering the Fourier transform, one can easily check

$$
\Delta^{\gamma / 2} p_{k}(t, x)=2^{k d} 2^{k \gamma} q_{k}\left(t, 2^{k} x\right) .
$$

Obviously, for all constants $a \geq 0$ and $c>0$,

$$
\sup _{x>0} x^{a} e^{-c x}<\infty .
$$


Recalling that $\hat{\Psi}(\xi)$ has the support in $B_{2} \backslash B_{1 / 2}$, differentiating $\hat{q}_{k}$ for $2 d$-times, and using (4.5), Assumptions 2.1(ii) and 2.10(iii), one can find positive constants $N$ and $c$ such that for all $(t, \xi) \in(0, T) \times \mathbf{R}^{d}$,

$$
\left|\hat{q}_{k}(t, \xi)\right|+\left|\Delta_{\xi}^{d} \hat{q}_{k}(t, \xi)\right| \leq N 1_{\left(2^{-1}, 2\right)}(|\xi|) \exp \left(-4 c \int_{0}^{t} \delta(s) d s \cdot\left|2^{k} \xi\right|^{2}\right) .
$$

Thus

$$
\begin{aligned}
\left|\left(1+|x|^{2 d}\right) q_{j}(t, x)\right| & =\left|\mathcal{F}^{-1}\left[\left(1+\Delta_{\xi}^{d}\right) \hat{q}_{k}(t, \xi)\right](x)\right| \\
& \leq N \sup _{|\xi| \in\left(2^{-1}, 2\right)}\left|\exp \left(-4 c \int_{0}^{t} \delta(s) d s \cdot\left|2^{k} \xi\right|^{2}\right)\right| \\
& \leq N \exp \left(-c \int_{0}^{t} \delta(s) d s \cdot 2^{2 k}\right)
\end{aligned}
$$

and

$$
\begin{aligned}
\left\|q_{k}(t, \cdot)\right\|_{L_{1}} & =\int_{|x| \leq 1}\left|q_{k}(t, x)\right| d x+\int_{|x| \geq 1}\left(|x|^{2 d}\left|q_{k}(t, x)\right|\right)|x|^{-2 d} d x \\
& \leq N \exp \left(-c \int_{0}^{t} \delta(s) d s \cdot 2^{2 k}\right) .
\end{aligned}
$$

Therefore by (4.4),

$$
\left\|\Delta^{\gamma / 2} p_{k}(t, \cdot)\right\|_{L_{1}} \leq N 2^{k \gamma} \exp \left(-c \int_{0}^{t} \delta(s) d s \cdot 2^{2 k}\right) .
$$

The lemma is proved.

Let $f \in \mathcal{S}\left(\mathbf{R}^{d}\right)$ and denote $f_{i}=\Delta_{i} f$. Note that Littlewood-Paley operators have the following orthogonal property:

$$
\begin{aligned}
& \Delta^{\gamma /(2 p)} p(t, \cdot) * f(x) \\
& =\left(\sum_{j \in \mathbb{Z}}^{\infty} \Delta^{\gamma /(2 p)} p_{j}(t, \cdot)\right) *\left(S_{0}(f)+\sum_{i=1}^{\infty} f_{i}\right)(x) \\
& =\sum_{j=-\infty}^{-1} \Delta^{\gamma /(2 p)} p_{j}(t, \cdot) * S_{0}(f)(x)+\sum_{i=1}^{\infty} \sum_{i-1 \leq j \leq i+1} \Delta^{\gamma /(2 p)} p_{j}(t, \cdot) * f_{i}(x) .
\end{aligned}
$$

This is because the intersection of the supports of $\hat{\Psi}\left(2^{-i} \xi\right)$ and $\hat{\Psi}\left(2^{-j} \xi\right)$ are nonempty only if $i-1 \leq j \leq i+1$. In particular,

$$
p(t, \cdot) * f(x)=p(t, \cdot) * \psi * S_{0}(f)(x)+\sum_{i=1}^{\infty} \sum_{i-1 \leq j \leq i+1} p_{j}(t, \cdot) * f_{i}(x),
$$

where

$$
\psi(x):=\mathcal{F}^{-1}\left[\sum_{j=-\infty}^{-1} \hat{\Psi}\left(2^{-j} \xi\right)\right](x) .
$$


Lemma 4.2. Let $T \in(0, \infty)$ and $\gamma \in\left[0, \frac{2}{\beta p}\right]$. Suppose that Assumptions 2.1 and 2.10 hold. Then for any $f \in \mathcal{S}\left(\mathbf{R}^{d}\right)$,

$$
\left\|\Delta^{\gamma / 2} \mathcal{T}_{t} f\right\|_{(0, T) \times \mathbf{R}^{d}}^{p} \leq N\left(\left\|S_{0}(f)\right\|_{L_{p}}^{p}+\sum_{j=1}^{\infty}\left\|f_{j}\right\|_{L_{p}}^{p}\right),
$$

where $N$ is a positive constant depending only on $d, p, \beta, N_{0}, \bar{N}_{0}, T$, and $\int_{0}^{t_{0}} \delta(s) d s$.

Proof. By (4.6) and Young's convolution inequality,

$$
\begin{aligned}
\int_{0}^{T}\left\|\Delta^{\gamma / 2} \mathcal{T}_{t} f(t, \cdot)\right\|_{L_{p}}^{p} d t \leq & \int_{0}^{T}\left(\sum_{j=-\infty}^{1}\left\|\Delta^{\gamma / 2} p_{j}(t, \cdot)\right\|_{L_{1}}\left\|S_{0}(f)\right\|_{L_{p}}\right)^{p} d t \\
& +\int_{0}^{T}\left(\sum_{i=1}^{\infty} \sum_{i-1 \leq j \leq i+1}\left\|\Delta^{\gamma / 2} p_{j}(t, \cdot)\right\|_{L_{1}}\left\|f_{i}\right\|_{L_{p}}\right)^{p} d t .
\end{aligned}
$$

By Lemma 4.1.

$$
\int_{0}^{T}\left(\sum_{j=-\infty}^{-1}\left\|\Delta^{\gamma / 2} p_{j}(t, \cdot)\right\|_{L_{1}}\left\|S_{0}(f)\right\|_{L_{p}}\right)^{p} d t \leq\left\|S_{0}(f)\right\|_{L_{p}}^{p}\left(\sum_{j=-\infty}^{-1} 2^{j \gamma}\right)^{p} .
$$

Obviously, if $\gamma>0$ then

$$
\left(\sum_{j=-\infty}^{-1} 2^{j \gamma}\right)^{p} \leq N
$$

For the case $\gamma=0$, we apply (4.7) and get

$$
\begin{aligned}
\int_{0}^{T}\left\|\mathcal{T}_{t} f(t, \cdot)\right\|_{L_{p}}^{p} d t \leq & \int_{0}^{T}\left(\|p(t, \cdot)\|_{L_{1}}\left\|\psi * S_{0}(f)\right\|_{L_{p}}\right)^{p} d t \\
& +\int_{0}^{T}\left(\sum_{i=1}^{\infty} \sum_{i-1 \leq j \leq i+1}\left\|p_{j}(t, \cdot)\right\|_{L_{1}}\left\|f_{i}\right\|_{L_{p}}\right)^{p} d t .
\end{aligned}
$$

Since $p(t, x)$ is the probability density function of the stochastic process $X_{t}$ introduced in (3.2),

$$
\|p(t, \cdot)\|_{L_{1}}=1 .
$$

Moreover, since $\psi(x)$ is contained in the Schwartz class,

$$
\left\|\psi * S_{0}(f)\right\|_{L_{p}} \leq N\left\|S_{0}(f)\right\|_{L_{p}}
$$

Combining (4.8) and (4.9), we have

$$
\int_{0}^{T}\left(\|p(t, \cdot)\|_{L_{1}}\left\|\psi * S_{0}(f)\right\|_{L_{p}}\right)^{p} d t \leq N \int_{0}^{T}\left\|S_{0}(f)\right\|_{L_{p}}^{p} d t .
$$

Therefore, it is sufficient to estimate the following term

$$
\int_{0}^{T}\left(\sum_{i=1}^{\infty} \sum_{i-1 \leq j \leq i+1}\left\|\Delta^{\gamma / 2} p_{j}(t, \cdot)\right\|_{L_{1}}\left\|f_{i}\right\|_{L_{p}}\right)^{p} d t .
$$


By Lemma 4.1 the above term is less than or equal to constant times of

$$
\mathcal{I}:=\int_{0}^{T}\left(\sum_{j=1}^{\infty} 2^{j \gamma} e^{-(c / 4) \int_{0}^{t} \delta(s) d s \cdot 2^{2 j}}\left\|f_{j}\right\|_{L_{p}}\right)^{p} d t
$$

To estimate $\mathcal{I}$, we consider the decomposition

$$
\mathcal{I}=\int_{t_{0}}^{T} \cdots d t+\int_{0}^{t_{0}} \cdots d t
$$

Define $\kappa_{0}:=\int_{0}^{t_{0}} \delta(s) d s>0$ and observe

$$
\sum_{j \geq 1} 2^{j \gamma q} e^{-c q \kappa_{0} 2^{2 j}}<\infty
$$

where $q:=p /(p-1)$. Therefore, by Hölder's inequality

$$
\begin{aligned}
& \int_{t_{0}}^{T}\left(\sum_{j=1}^{\infty} 2^{j \gamma} e^{-c \int_{0}^{t} \delta(s) d s \cdot 2^{2 j}}\left\|f_{j}\right\|_{L_{p}}\right)^{p} d t \leq T\left(\sum_{j=1}^{\infty} 2^{j \gamma} e^{-c \kappa_{0} \cdot 2^{2 j}}\left\|f_{j}\right\|_{L_{p}}\right)^{p} \\
& \leq T\left(\sum_{j=1}^{\infty} 2^{j \gamma q} e^{-c q 2^{2 j}}\right)^{1 / q} \sum_{j=1}^{\infty}\left\|f_{j}\right\|_{L_{p}}^{p} \leq N \sum_{j=1}^{\infty}\left\|f_{j}\right\|_{L_{p}}^{p} .
\end{aligned}
$$

Thus it only remains to show

$$
\int_{0}^{t_{0}}\left(\sum_{i=1}^{\infty} 2^{j \gamma} e^{-(c / 4) \int_{0}^{t} \delta(s) d s \cdot 2^{2 j}}\left\|f_{j}\right\|_{L_{p}}\right)^{p} d t \leq N \sum_{i=1}^{\infty}\left\|f_{j}\right\|_{L_{p}}^{p} d t .
$$

Note that

$$
\int_{0}^{t_{0}}\left(\sum_{j=1}^{\infty} 2^{j \gamma} e^{-(c / 4) \int_{0}^{t} \delta(s) d s \cdot 2^{2 j}}\left\|f_{j}\right\|_{L_{p}}\right)^{p} d t \leq 2^{p}\left(\mathcal{I}_{1}+\mathcal{I}_{2}\right)
$$

where

$$
\mathcal{I}_{1}=\int_{0}^{t_{0}}\left(\sum_{2^{2 j} \int_{0}^{t} \delta(s) d s \leq 1} 2^{j \gamma} e^{-c \int_{0}^{t} \delta(s) d s \cdot 2^{2 j}}\left\|f_{j}\right\|_{L_{p}}\right)^{p} d t
$$

and

$$
\mathcal{I}_{2}=\int_{0}^{t_{0}}\left(\sum_{2^{2 j} \int_{0}^{t} \delta(s) d s>1} 2^{j \gamma} e^{-c \int_{0}^{t} \delta(s) d s \cdot 2^{2 j}}\left\|f_{j}\right\|_{L_{p}}\right)^{p} d t .
$$


First we estimate $\mathcal{I}_{1}$. Observe that $2^{2 j} \int_{0}^{t} \delta(s) d s \leq 1$ if and only if $j \leq \log _{2}\left(\int_{0}^{t} \delta(s) d s\right)^{-1 / 2}$.

Using Hölder's inequality and Fubini's theorem (also use $1=2^{\frac{j}{\beta p}} 2^{-\frac{j}{\beta p}}$ ), we get (recall $q=p /(p-1))$,

$$
\begin{aligned}
\mathcal{I}_{1} & \leq \int_{0}^{t_{0}}\left(\sum_{\int_{0}^{t} \delta(s) d s \cdot 2^{2 j} \leq 1} 2^{\frac{q j}{\beta p}}\right)^{p-1} \sum_{\int_{0}^{t} \delta(s) d s \cdot 2^{2 j} \leq 1}^{\infty} 2^{\left(\gamma p-\frac{1}{\beta}\right) j}\left\|f_{j}\right\|_{L_{p}}^{p} d t \\
& \leq N \int_{0}^{t_{0}}\left(\int_{0}^{t} \delta(s) d s\right)^{-\frac{1}{2 \beta}} \sum_{\int_{0}^{t} \delta(s) d s \cdot 2^{2 j} \leq 1} 2^{\left(\gamma p-\frac{1}{\beta}\right) j}\left\|f_{j}\right\|_{L_{p}}^{p} d t \\
& \leq N \sum_{j=1}^{\infty} 2^{\left(\gamma p-\frac{1}{\beta}\right) j}\left\|f_{j}\right\|_{L_{p}}^{p} \int_{0}^{t_{0}} 1_{\int_{0}^{t} \delta(s) d s \cdot 2^{2 j} \leq 1}(t)\left(\int_{0}^{t} \delta(s) d s\right)^{-\frac{1}{2 \beta}} d t .
\end{aligned}
$$

Due to Assumption 2.10(ii),

$$
\begin{aligned}
& \int_{0}^{t_{0}} 1_{\int_{0}^{t} \delta(s) d s \cdot 2^{2 j} \leq 1}(t)\left(\int_{0}^{t} \delta(s) d s\right)^{-\frac{1}{2 \beta}} d t \\
& \leq \sum_{m=0}^{\infty}\left|\left\{t \in\left(0, t_{0}\right): 2^{-2(j+m+1)} \leq \int_{0}^{t} \delta(s) d s \leq 2^{-2(j+m)}\right\}\right| 2^{\frac{j+m+1}{\beta}} \\
& \leq N 2^{-\frac{j}{\beta}} \sum_{m=0}^{\infty} 2^{-\frac{m}{\beta}} \leq N 2^{-\frac{j}{\beta}} .
\end{aligned}
$$

Therefore,

$$
\mathcal{I}_{1} \leq N \sum_{j=1}^{\infty} 2^{\left(\gamma p-\frac{2}{\beta}\right) j}\left\|f_{j}\right\|_{L_{p}}^{p} \leq N \sum_{j=1}^{\infty}\left\|f_{j}\right\|_{L_{p}}^{p}
$$

Next we estimate $\mathcal{I}_{2}$. For $b \geq 0$, using the equality $1=2^{b j} 2^{-b j}$ and the Hölder inequality, we get

$\mathcal{I}_{2} \leq \int_{0}^{t_{0}}\left(\sum_{\int_{0}^{t} \delta(s) d s \cdot 2^{2 j}>1} 2^{(\gamma+b) q j} e^{-c q \int_{0}^{t} \delta(s) d s \cdot 2^{2 j}}\right)^{p-1} \sum_{\int_{0}^{t} \delta(s) d s \cdot 2^{2 j}>1}^{\infty} 2^{-b p j}\left\|f_{j}\right\|_{L_{p}}^{p} d t$.

By (4.5), for any positive constant $a>\frac{\gamma+b}{2}$,

$$
e^{-c q \int_{0}^{t} \delta(s) d s \cdot 2^{2 j}} \leq N 2^{-2 a q j}\left(\int_{0}^{t} \delta(s) d s\right)^{-a q}
$$


Thus by Assumption 2.10(ii),

$$
\begin{aligned}
\mathcal{I}_{2} \leq & \int_{0}^{t_{0}}\left(\left(\int_{0}^{t} \delta(s) d s\right)^{-a q} \sum_{\int_{0}^{t} \delta(s) d s \cdot 2^{2 j}>1} 2^{(\gamma+b-2 a) q j}\right)^{p-1} \\
& \times \sum_{\int_{0}^{t} \delta(s) d s \cdot 2^{2 j}>1}^{\infty} 2^{-b p j}\left\|f_{j}\right\|_{L_{p}}^{p} d t \\
\leq & N \int_{0}^{t_{0}}\left(\int_{0}^{t} \delta(s) d s\right)^{-\frac{(\gamma+b) p}{2}} \sum_{j}^{\infty} 2^{-b p j}\left\|f_{j}\right\|_{L_{p}}^{p} d t \\
\leq & N \sum_{j=1}^{\infty} 2^{-b p j}\left\|f_{j}\right\|_{L_{p}}^{p} \int_{0}^{t_{0}}\left(\int_{0}^{t} \delta(s) d s\right)^{-\frac{(\gamma+b) p}{2}} 1_{\int_{0}^{t} \delta(s) d s \cdot 2^{2 j}>1}(t) d t \\
\leq & N \sum_{j=1}^{\infty} \sum_{m=0}^{\infty} 2^{\gamma p j} 2^{-(\gamma+b) p m}\left\|f_{j}\right\|_{L_{p}}^{p} \\
& \times\left|\left\{t \in\left[0, t_{0}\right]: 2^{-2(j-m)} \leq \int_{0}^{t} \delta(s) d s \leq 2^{-2(j-m-1)}\right\}\right| \\
\leq & N \sum_{m=0}^{\infty} 2^{\left(\frac{2}{\beta}-(\gamma+b) p\right) m} \sum_{j=1}^{\infty} 2^{\left(\gamma p-\frac{2}{\beta}\right) j}\left\|f_{j}\right\|_{L_{p}}^{p}
\end{aligned}
$$

Take $b=\frac{4}{\beta p}$. Then

$$
\frac{2}{\beta}-(\gamma+b) p<0
$$

Therefore, from (4.10), we have

$$
\mathcal{I}_{2} \leq N \sum_{j=1}^{\infty} 2^{\left(\gamma p-\frac{2}{\beta}\right) j}\left\|f_{j}\right\|_{L_{p}}^{p} \leq N \sum_{j=1}^{\infty}\left\|f_{j}\right\|_{L_{p}}^{p} .
$$

The lemma is proved.

We continue the proof of the theorem and assume $u_{0} \in \mathcal{S}\left(\mathbf{R}^{d}\right)$. Define $v=$ $(1-\Delta)^{(1-1 /(\beta p))} u$ and $v_{0}:=(1-\Delta)^{(1-1 /(\beta p))} u_{0}$. Then obviously $v_{0} \in \mathcal{S}\left(\mathbf{R}^{d}\right)$ and $v$ satisfies

$$
v_{t}=a^{i j}(t) v_{x^{i} x^{j}}, \quad t \in(0, T) ; \quad v(0, \cdot)=v_{0} .
$$

Thus by (4.2) and Lemma 4.2.

$$
\|v\|_{\mathbb{H}_{p}^{2 /(\beta p)}} \leq N\left(\|v\|_{\mathbb{L}_{p}}+\left\|\Delta^{1 /(\beta p)} v\right\|_{\mathbb{L}_{p}}\right) \leq N\left(\left\|S_{0}\left(v_{0}\right)\right\|_{L_{p}}+\sum_{j=1}^{\infty}\left\|\left(v_{0}\right)_{j}\right\|_{L_{p}}^{p}\right),
$$

where the first inequality can be easily induced from the classical multiplier theorem (e.g. [5, Theorem 5.2.7]). Since the operator $(1-\Delta)^{(1-1 /(\beta p))}$ is an isometry from $H^{2 /(\beta p)}\left(\operatorname{resp} . B_{p}^{0}\right)$ to $H_{p}^{2}\left(\operatorname{resp} . B_{p}^{2(1-1 /(\beta p))}\right)$ (see [1, Theorem 6.2.7]), it follows from (4.11) that

$$
\|u\|_{\mathbb{H}_{p}^{2}(T)} \leq N\left\|u_{0}\right\|_{B_{p}^{2(1-1 /(\beta p))}}
$$


For general $u_{0}$, it is enough to apply a standard approximation based on (4.12). The theorem is proved.

\section{Acknowledgement}

We are sincerely grateful to the referee. Especially, we could considerably enhance Theorem 2.7 due to the referee.

\section{REFERENCES}

[1] J. Bergh and J. Löfström. Interpolation spaces. an introduction. 1976.

[2] S. Fornaro, G. Metafune, D. Pallara, and R. Schnaubelt. Degenerate operators of tricomi type in lp-spaces and in spaces of continuous functions. Journal of Differential Equations, 252(2):1182-1212, 2012.

[3] M. Freidlin. Smoothness of solutions of degenerating elliptic equations. Technical report, DTIC Document, 1969.

[4] M. Gerencsér, I. Gyöngy, and N. Krylov. On the solvability of degenerate stochastic partial differential equations in sobolev spaces. Stochastic Partial Differential Equations: Analysis and Computations, 3(1):52-83, 2015.

[5] L. Grafakos. Classical Fourier analysis, volume 249. Springer, 2008

[6] L. Grafakos. Modern Fourier Analysis, volume 250. Springer, 2009.

[7] K.-H. Kim. Sobolev space theory of parabolic equations degenerating on the boundary of $C^{1}$-domains. Communications in Partial Differential Equations, 32(8):1261-1280, 2007.

[8] K.-H. Kim and K. Lee. On the heat diffusion starting with degeneracy. Journal of Differential Equations, 262(3):2722-2744, 2017.

[9] J. J. Kohn and L. Nirenberg. Degenerate elliptic-parabolic equations of second order. Communications on Pure and Applied Mathematics, 20(4):797-872, 1967.

[10] N. V. Krylov and B. Rozovskii. Characteristics of degenerating second-order parabolic ito equations. Journal of Soviet Mathematics, 32(4):336-348, 1986.

[11] N. V. Krylov. Introduction to the theory of diffusion processes, volume 96. American Mathematical Society, 1995.

[12] N. V. Krylov. Lectures on Elliptic and Parabolic Equations in Sobolev Spaces, volume 96. American Mathematical Society Providence, RI, 2008.

[13] N. V. Krylov. A parabolic Littlewood-Paley inequality with applications to parabolic equations Topological Methods in Nonlinear Analysis, Journal of the Juliuss Schauder Center, 4 (1994), 355-364.

[14] O.A. Oleinik, Alcuni risultati sulle equazioni lineari e quasi lineari ellitticoparaboliche a derivate parziali del secondo ordine, (Italian) Atti Accad. Naz. Lincei Rend. Cl. Sci. Fis. Mat. Natur., (8) 40, (1966), 775-784.

[15] O.A. Oleinik, On the smoothness of solutions of degenerating elliptic and parabolic equations, Dokl. akad. Nauk SSSR, 163 (1965), 577-580 in Russian; English translation in Soviet Mat. Dokl, 6 (1965), no. 3, 972-976.

[16] O. A. Oleïnik and E. V. Radkevič, Second order equations with nonnegative characteristic form, Mathematical Analysis, 1969, pp. 7-252. (errata insert) Akad. Nauk SSSR, Vsesojuzn. Inst. Naučn. i Tehn. Informacii, Moscow, 1971 in Russian; English translation: Plenum Press, New York-London, 1973.

[17] O. A. Olë̈nik and E. V. Radkevich, Second Order Equations with Nonnegative Characteristic Form, AMS, Providence 1973.

[18] O .A. Oleinik. Second-order equations with nonnegative characteristic form. Springer Science \& Business Media, 2012.

[19] D. Stroock and S. Varadhan. On degenerate elliptic-parabolic operators of second order and their associated diffusions. Communications on Pure and Applied Mathematics, 25(6):651$713,1972$. 
Department of mathematics, Korea university, 1 anam-Dong Sungbuk-Gu, Seoul, South Korea 136-701

E-mail address: waldoo@korea.ac.kr

Department of mathematics, Korea university, 1 AnAm-Dong Sungbuk-Gu, SeOul, South KOREA 136-701

E-mail address: kyeonghun@korea.ac.kr 